\title{
Consistency of children's dietary choices: annual repeat measures from 5 to 13 years (EarlyBird 49)
}

\author{
Alissa E. Frémeaux, Joanne Hosking, Brad S. Metcalf, Alison N. Jeffery, Linda D. Voss and \\ Terence J. Wilkin* \\ Department of Endocrinology and Metabolism, Peninsula College of Medicine and Dentistry, University Medicine, Level 7 , \\ Derriford Hospital, Plymouth PL6 8DH, UK
}

(Received 14 September 2010 - Revised 17 January 2011 - Accepted 21 January 2011 - First published online 10 May 2011)

\begin{abstract}
The objective of the present study was to explore the consistency of dietary choices made by children as they grow up. The dietary habits of 342 healthy children were reported annually from 5 to 13 years on a forty-five-item FFQ and analysed by factor analysis. The same two principal dietary patterns - 'Healthy' and 'Unhealthy' - emerged each year, and their consistency was assessed using Tucker's congruence coefficient $(\varphi)$. Individual dietary $z$-scores for both of these patterns were then calculated every year for each child, and their consistency was measured by Pearson's correlation coefficient $(r)$. Linear mixed-effects modelling was used to investigate individual trends and to quantify reliability of the individual dietary $z$-scores. Dietary patterns were moderately consistent and systematic over time $\left(0 \cdot 65 \leq \varphi_{\text {Healthy }} \leq 0.76\right.$; $\left.0.62 \leq \varphi_{\text {Unhealthy }} \leq 0.78\right)$. Individual choices were also consistent year-on-year $\left(0.64 \leq r_{\text {Healthy }} \leq 0.71 ; 0.57 \leq r_{\text {Unhealthy }} \leq 0.68\right)$. Reliability rose from $70 \%$ with a single measure to over $90 \%$ with four consecutive measures. The quality of diet diminished over time in $29 \%$ of the children and improved in only 14\%. Dietary habits appear to be set early and seldom improve spontaneously.
\end{abstract}

Key words: Dietary choices: Consistency: Longitudinal studies: EarlyBird

Obesity is arguably the biggest threat to health in the twentyfirst century. Obesity is believed to underlie the rising incidence of diabetes, heart disease and cancer $^{(1)}$, and a better understanding of its origins is likely to prove key to their prevention. The dietary habits of adults are influenced by those acquired during childhood ${ }^{(2,3)}$, so that the epidemiology of children's nutrition may help to understand the rise in obesity generally.

Rigorous assessment of dietary choice is needed to understand its association with obesity but can prove difficult in free-living children. Accordingly, most studies have relied on a single measurement in time, although cross-sectional analysis can only reveal association, which is not always causal. Some have repeated the measurement to assess change, but multiple measures are needed to derive a trend - and the trend is essential to establish consistency and interaction over time. Longitudinal studies of dietary trends are scarce in children, and multivariate approaches, such as factor analysis to reveal dietary patterns ${ }^{(4)}$, are rarer still. Pattern analysis is arguably preferable to the traditional examination of single food items, which cannot take account of food combinations or evaluate changes in their structure of a diet over time.

To date, no study has repeatedly monitored the consistency of dietary choice in a single cohort of children over the long term. In the present study, we record the dietary patterns and their consistency in a large cohort of contemporary children, and report on their trends over 8 years. We wanted to know how contemporary children assemble the foods they choose to eat, how stable the food clusters remain with time and to what extent individual children preserve their early choices over the years.

\section{Methods}

\section{Participants}

The study is based on data from the ongoing EarlyBird Diabetes Study, an observational prospective cohort study of healthy children from Plymouth (UK). The protocol has been described in detail elsewhere ${ }^{(5)}$. The study recruited 307 children (170 boys and 137 girls) at the age of 5 years (4.9 (SD 0.3) years) in 2000 and another forty children in 2004 (age 9 years; 8.8 (SD 0.3) years) to establish sex balance and make up for first-year losses. Accordingly, data will be presented from 5 to 9 years $(n$ 307) and from 9 to 13 years ( $n$ 347). The additional children represented $12 \%$ of the total, and there were no differences from those recruited at 9 years in any of the several measures made by the study.

*Corresponding author: T. J. Wilkin, fax +44 1752 792471, email t.wilkin@pms.ac.uk 
Importantly, the age range of the cohort was narrow ( $\mathrm{SD} \pm 3$ months) to best resolve age-related change. Parents or guardians of the child were asked to complete a FFQ annually, as part of a review which also included fasting blood samples, body composition (dual-energy X-ray absorptiometry), physical activity (accelerometry) and resting energy expenditure (gas exchange).

The present study was conducted according to the guidelines laid down in the Declaration of Helsinki, and all procedures involving human subjects/patients were approved by the Plymouth Local Research Ethics Committee (no. 1224) in 1999. Informed written consent and verbal assent were jointly obtained from parents and children, respectively.

\section{Dietary assessment}

At each annual visit, the same forty-five-item (or food group) FFQ was completed by the parents on behalf of their children, in order to profile habitual intakes of food, beverages and cooking fat during the previous 12 months. School lunches and other food eaten outside of the home were included. The questionnaire was adapted from an earlier version, designed for use in children and validated against fourteen daily recalls using the same food list ${ }^{(6)}$. The authors concluded that 'The questionnaire proved a valid instrument for classifying children into broad patterns of consumption in an epidemiological study...' Retaining the same format, we added potato crisps, low-energy soft drinks and low-fat spreads to reflect the changing dietary intake of contemporary children.

Each item or food group was allocated to one of ten frequency categories ranging from 'never', 'once per month', 'once per fortnight' and ' $1 \mathrm{~d}$ a week' to ' $7 \mathrm{~d}$ a week'. The selected frequency choice for each item was converted into a weekly intake for analysis. An additional question asked whether the child had taken any food supplements in the preceding month, which ones (e.g. vitamins, minerals, tonics) and with what frequency. Where possible, items left blank were imputed as the mean of the frequencies reported the previous year and the following year. Imputation is particularly valuable in factor analysis, which does not permit missing values.

\section{Statistical analysis}

Factor analysis is a data-reduction technique used to uncover the underlying structure of a large set of variables. It was performed in the present study to reveal the principal dietary patterns of the children on the basis of shared covariance among the forty-five food items. Food items were tested for normal distribution, and some were removed from further analysis if not complying with the test. The number of factors retained was decided after inspection of a scree plot, which provides a graphical representation of eigenvalues ${ }^{(7)}$. The best 'statistical' solution suggested a three-factor solution. The third factor (milk) did explain a certain proportion of the variance in the data (because almost every child drinks milk) but related only to the type of milk consumed, which tended to be mutually exclusive, so we opted to focus on the first two factors. Factors were then rotated with oblique (Promax) rotation to enhance interpretability while allowing for potential correlation between factors. Each factor was made up from food items with a loading higher than $0 \cdot 30$, which was considered as significantly contributing to the factor (dietary pattern). Factor loadings reflect the degree of affiliation of each food item with a factor. For each child, we then calculated dietary factor $z$-scores by summing all food frequency of intake (10-point), weighted by their factor loadings. These scores reflect the degree to which each child conformed to a given dietary pattern. Dietary patterns were similar for boys and girls, therefore analyses were carried out on both sexes together.

Year-on-year consistency of the dietary patterns of the cohort as a whole was quantified by Tucker's congruence coefficient $(\varphi)^{(8)}$, which measures the similarity between two factor solutions. Pearson's correlation coefficient was used to assess the consistency of each child's dietary factor scores, from 5 to 13 years. Linear mixed-effects modelling with random intercepts and random slopes was used on the dietary $z$-scores derived for the two factors retained, in order to assess the reliability of the measure and identify trends from 5 to 13 years. All analyses were performed using the freeware R 2.8.1 (www.r-project.org/).

\section{Results}

The characteristics of the subjects are described in Table 1.

\section{Sample attrition and response rates}

During the 8 years of follow-up, two children were diagnosed with coeliac disease and three with diabetes. They were excluded from the analysis. Of the remaining 302 children, forty-four recruited at the age of 5 years had left the study by 9 years, leaving a sample size of 258 children. By 13 years of age, 276 ( $81 \%$ ) of the total 342 children recruited were still in the study. Based on these numbers, response rates for the FFQ ranged from 94 to $98 \%$ between 5 and 13 years, with a mean of $96 \cdot 4 \%$. Non-response included nonreturn of the questionnaire (1.8\%), incorrect completion (1.5\%) or blank return (0.3\%). Of the 2470 questionnaires retained for analysis, fewer than $1 \%$ of the data fields were imputed as a consequence of omission.

\section{Factor analysis}

The factor analyses revealed the same three major factors every year. The first - characterised by high-frequency intake of 'elemental' foods such as vegetables, fruits, fish, chicken, eggs, pasta, rice, high-fibre cereals, brown bread and olive oil - was labelled 'Healthy'. The second - characterised by high-frequency intake of energy-dense 'processed' foods such as crisps, chips, sausages, processed meats, white bread, sweets and chocolate bars, biscuits, cakes, soft drinks and vegetable oil - was labelled 'Unhealthy'. The third factor reflected the type of milk consumed (full fat or skimmed). This three-factor solution explained $16-18 \%$ of the total variance in the data between 5 and 13 years. We analysed only the first two factors, as the third incorporated only 
Table 1. Summary characteristics of the study population at the age of 5 and 9 years (incorporating new recruits) (Mean values, standard deviations, number of participants and percentages)

\begin{tabular}{|c|c|c|c|c|c|c|c|c|}
\hline & \multicolumn{4}{|c|}{5 years } & \multicolumn{4}{|c|}{9 years } \\
\hline & \multicolumn{2}{|c|}{ Boys ( $n$ 170) } & \multicolumn{2}{|c|}{ Girls (n 137) } & \multicolumn{2}{|c|}{ Boys ( $n$ 146) } & \multicolumn{2}{|c|}{ Girls $(n 146)$} \\
\hline & $n$ & $\%$ & $n$ & $\%$ & $n$ & $\%$ & $n$ & $\%$ \\
\hline \multicolumn{9}{|l|}{ Age (years) } \\
\hline Mean & \multicolumn{2}{|c|}{4.9} & \multicolumn{2}{|c|}{4.9} & \multicolumn{2}{|c|}{$8 \cdot 9$} & \multicolumn{2}{|c|}{$8 \cdot 8$} \\
\hline SD & \multicolumn{2}{|c|}{0.3} & \multicolumn{2}{|c|}{0.3} & & & \multicolumn{2}{|c|}{0.3} \\
\hline \multicolumn{9}{|l|}{ Ethnicity } \\
\hline White & 167 & 98 & 135 & 99 & 143 & 98 & 142 & 97 \\
\hline Non-White & 3 & 2 & 2 & 1 & 3 & 2 & 4 & 3 \\
\hline \multicolumn{9}{|l|}{ Socio-economic status } \\
\hline Deprived & 24 & 14 & 33 & 24 & 15 & 10 & 23 & 16 \\
\hline Non-deprived & 146 & 86 & 104 & 76 & 131 & 90 & 123 & 84 \\
\hline \multicolumn{9}{|l|}{ Weight category } \\
\hline Normal weight (<91st centile) & 145 & 85 & 111 & 81 & 115 & 79 & 112 & 77 \\
\hline Overweight/obese ( $\geq 91$ st centile) & 25 & 15 & 26 & 19 & 31 & 21 & 34 & 23 \\
\hline
\end{tabular}

two relevant items, which tended to be mutually exclusive. Among the forty-five items reported, three (liver or kidney, cottage cheese and ghee) were excluded from the analysis because of their very low consumption. Factor-loading matrices from 5 to 13 years for the remaining two factors (Healthy and Unhealthy) are presented in Table 2. Food items with high loadings reflect a high contribution to the corresponding factor.

\section{Consistency of the dietary patterns - cohort}

Tucker's congruence coefficients (by definition $0 \leq \varphi \leq 1$ ) were calculated for the Healthy and Unhealthy factors from 5 to 13 years. A congruence coefficient close to 1 would indicate a strong similarity between factor solutions year-byyear. Matrices showing all paired comparisons across the nine annual time points are presented in Table 2. Analysis revealed a systematic and moderately high degree of similarity in the factor solutions between 5 and 13 years for both Healthy $\left(0.65 \leq \varphi_{\text {Healthy }} \leq 0.76\right)$ and Unhealthy $(0.62 \leq$ $\left.\varphi_{\text {Unhealthy }} \leq 0 \cdot 78\right)$ patterns.

\section{Consistency and reliability of dietary z-scores - individuals}

Dietary $z$-scores were created for the Healthy and Unhealthy factors, and for each child. Since both patterns were consistent from 5 to 13 years, we assessed the consistency of the individual dietary $z$-scores over time. Pearson's correlation coefficients revealed close tracking (Tables 3 and 4). Year-on-year correlations ranged from 0.64 to 0.71 for the Healthy $z$-scores, and between 0.57 and 0.68 for the Unhealthy $z$-scores (all $P<0 \cdot 001$ ). When comparing years further apart, correlations were inevitably attenuated $\left(r_{\text {Healthy }}=0.46\right.$ and $r_{\text {Unhealthy }}=0.33$ between 5 and 13 years), reflecting gradual individual changes with time.

The use of a linear mixed-effects model (with random intercepts and random slopes) allowed us to assess the intra- and inter-subject variance of the dietary $z$-scores, by taking advantage of all time points available for each child (mean $7 \cdot 2$ ). Estimates of the mean reliability of the measures, as a function of the number of time points available (Fig. 1), revealed that at least four annual measurements were required to achieve a minimum of $90 \%$ reliability on both dietary $z$-scores.

Analysis of the trend between 5 and 13 years (based on 302 children), using the slopes extracted with the linear mixedeffects model, revealed that only $14 \%$ of the children recruited at 5 years of age record both a higher $z$-score at 13 years on the Healthy factor and a lower $z$-score on the Unhealthy factor. Approximately $29 \%$ of the children showed the reverse - a higher score on the unhealthy with a lower score on the healthy diet, while the others recorded an increase $(29 \%)$ or a decrease $(28 \%)$ on both dietary factors.

\section{Discussion}

Annual FFQ data analysed by factor analysis revealed two main dietary patterns in a cohort of healthy contemporary children. The first, labelled 'Healthy', consisted of largely 'elemental' foods, while the second, labelled 'unhealthy', was characterised by energy-dense 'processed' foods. Tucker's congruence coefficients confirmed consistency in the factor solutions for the cohort overall - i.e. the two food groups and their content turned up in much the same form from year to year. Consistency overall is pre-requisite to the analysis of individual dietary $z$-scores. Correlation coefficients among individuals were high when comparing successive years but weakened over longer time intervals, suggesting a change in children's dietary choices over time. We then quantified the within- and between-subject variance in the dietary $z$-scores over the nine time points, assessed their reliability and looked for individual trends using mixed-effects modelling. A minimum of four measurements was needed to achieve $90 \%$ reliability, a benchmark that cannot be obtained by single measures.

Until now, most studies exploring the consistency of dietary intake have relied on one ${ }^{(9,10)}$, or in some cases two, followup measurement, and many of these were limited to nutrients 
Table 2. Factor-loading matrices for the two major dietary patterns identified by factor analysis, from 5 to 13 years

\begin{tabular}{|c|c|c|c|c|c|c|c|c|c|c|c|c|c|c|c|c|c|c|c|c|}
\hline & \multicolumn{2}{|c|}{5 years $(n 283)$} & \multicolumn{2}{|c|}{6 years $(n 271)$} & \multicolumn{2}{|c|}{7 years $(n 266)$} & \multicolumn{2}{|c|}{8 years $(n 253)$} & \multicolumn{2}{|c|}{9 years $(n 252)$} & \multicolumn{2}{|c|}{9 years $(n 292)$} & \multicolumn{2}{|c|}{10 years $(n 290)$} & \multicolumn{2}{|c|}{11 years $(n 280)$} & \multicolumn{2}{|c|}{12 years $(n 260)$} & \multicolumn{2}{|c|}{13 years $(n 261)$} \\
\hline & $\mathrm{H}$ & UnH & $\mathrm{H}$ & UnH & $\mathrm{H}$ & UnH & $\mathrm{H}$ & UnH & $\mathrm{H}$ & UnH & $\mathrm{H}$ & UnH & $\mathrm{H}$ & UnH & $\mathrm{H}$ & UnH & $\mathrm{H}$ & UnH & $\mathrm{H}$ & UnH \\
\hline Beef & & 0.14 & & 0.24 & 0.21 & $0.31^{*}$ & 0.12 & 0.28 & 0.15 & 0.26 & 0.16 & 0.28 & & 0.29 & 0.17 & $0.30^{*}$ & 0.27 & 0.24 & 0.16 & 0.15 \\
\hline Lamb & 0.26 & 0.13 & 0.16 & 0.18 & 0.27 & & & & 0.19 & 0.17 & 0.17 & 0.14 & 0.11 & 0.19 & 0.15 & 0.16 & 0.22 & 0.13 & 0.21 & \\
\hline Pork & 0.16 & 0.15 & 0.12 & 0.17 & & 0.26 & 0.15 & 0.13 & 0.21 & 0.23 & 0.24 & 0.26 & 0.12 & 0.26 & & 0.13 & 0.14 & 0.22 & 0.12 & 0.16 \\
\hline Sausages & & $0.44^{*}$ & & $0.31^{*}$ & & 0.25 & -0.12 & 0.18 & & 0.24 & & 0.22 & & 0.21 & 0.11 & $0.40^{*}$ & & 0.24 & & $0.31^{\star}$ \\
\hline Sliced processed meats & & $0.30^{*}$ & & 0.26 & & $0.32^{*}$ & & $0.32^{*}$ & & $0.33^{*}$ & & 0.29 & & $0.33^{*}$ & & $0.37^{\star}$ & & $0.43^{\star}$ & & 0.29 \\
\hline Chicken & 0.25 & -0.11 & 0.25 & & 0.14 & & 0.28 & 0.15 & $0.33^{*}$ & 0.14 & $0.33^{\star}$ & 0.18 & 0.27 & & $0.36^{*}$ & & & & 0.14 & \\
\hline $\begin{array}{l}\text { Fish fingers/fish cakes/ } \\
\text { fish in batter }\end{array}$ & 0.13 & 0.25 & 0.19 & 0.20 & & 0.12 & & & & 0.22 & & 0.22 & & & & 0.11 & 0.19 & & 0.21 & 0.16 \\
\hline Oily fish & $0.42^{*}$ & & $0.36^{*}$ & -0.11 & 0.20 & -0.17 & $0.30^{*}$ & -0.16 & 0.29 & -0.15 & 0.27 & -0.17 & 0.25 & -0.24 & $0.38^{*}$ & -0.13 & 0.21 & -0.23 & 0.16 & -0.21 \\
\hline Other fish & $0.48^{\star}$ & -0.14 & $0.42^{*}$ & & 0.18 & -0.17 & 0.16 & -0.22 & 0.27 & & 0.27 & & 0.24 & -0.13 & 0.28 & -0.10 & 0.27 & -0.24 & 0.25 & $-0.31^{*}$ \\
\hline Cheese & 0.25 & 0.15 & 0.23 & & 0.27 & & $0.33^{*}$ & 0.11 & 0.21 & & 0.20 & & 0.16 & & 0.22 & & 0.24 & & 0.25 & \\
\hline $\begin{array}{l}\text { Skimmed/semi-skimmed } \\
\text { milk }\end{array}$ & 0.15 & & & & & 0.21 & & 0.15 & & 0.15 & & 0.16 & & & & 0.13 & & 0.12 & & \\
\hline Full-fat milk & & & & & & -0.14 & 0.13 & & & -0.14 & & -0.18 & & & & & 0.12 & & 0.11 & \\
\hline Yogurt & & 0.13 & 0.13 & 0.22 & 0.15 & 0.22 & & 0.27 & & 0.14 & 0.13 & 0.12 & & 0.12 & & & & 0.21 & 0.23 & 0.11 \\
\hline Eggs & 0.21 & & $0.38^{*}$ & & $0.44^{*}$ & 0.14 & $0.35^{*}$ & & $0.46^{*}$ & 0.18 & $0.42^{\star}$ & 0.11 & 0.18 & & $0.35^{*}$ & & 0.26 & & $0.42^{*}$ & \\
\hline $\begin{array}{l}\text { Low-fibre breakfast } \\
\text { cereals }\end{array}$ & -0.10 & 0.20 & & 0.16 & & 0.25 & -0.12 & 0.16 & -0.13 & 0.14 & -0.18 & 0.13 & -0.11 & 0.13 & & 0.23 & & 0.24 & & 0.20 \\
\hline $\begin{array}{l}\text { High-fibre breakfast } \\
\text { cereals }\end{array}$ & 0.17 & -0.13 & 0.27 & & 0.24 & -0.13 & 0.29 & & 0.22 & & 0.19 & -0.12 & 0.16 & -0.17 & $0.36^{*}$ & & $0.30^{*}$ & & $0.38^{*}$ & -0.18 \\
\hline Pasta & $0.38^{*}$ & & $0.37^{\star}$ & & $0.43^{*}$ & & $0.31^{*}$ & -0.13 & $0.39^{*}$ & & $0.37^{\star}$ & & & -0.20 & $0.33^{*}$ & & 0.25 & & 0.24 & \\
\hline Rice & $0.35^{\star}$ & -0.11 & $0.40^{\star}$ & -0.11 & $0.41^{*}$ & -0.23 & $0.34^{*}$ & -0.13 & $0.48^{*}$ & & $0.45^{\star}$ & & 0.23 & & $0.46^{*}$ & & 0.25 & -0.12 & 0.29 & \\
\hline Sweet biscuits & & $0.51^{\star}$ & & $0.57^{\star}$ & & $0.65^{\star}$ & & $0.50^{*}$ & & $0.48^{\star}$ & & $0.45^{\star}$ & & $0.47^{\star}$ & & $0.43^{*}$ & & $0.52^{*}$ & & $0.55^{*}$ \\
\hline Cakes/scones & & $0.37^{*}$ & 0.17 & $0.50^{*}$ & 0.12 & $0.37^{*}$ & 0.15 & $0.37^{*}$ & & $0.48^{*}$ & & $0.45^{*}$ & 0.15 & $0.48^{*}$ & & $0.36^{*}$ & & $0.35^{*}$ & 0.22 & 0.28 \\
\hline Sweets/chocolate bars & -0.20 & $0.47^{\star}$ & -0.15 & $0.51^{\star}$ & & $0.52^{*}$ & 0.11 & $0.59^{*}$ & & $0.52^{*}$ & & $0.52^{*}$ & & $0.45^{*}$ & -0.16 & $0.45^{*}$ & & $0.46^{*}$ & & $0.51^{\star}$ \\
\hline Crisps & & $0.55^{*}$ & & $0.53^{*}$ & -0.17 & $0.51^{*}$ & & $0.65^{*}$ & & $0.62^{\star}$ & & $0.62^{*}$ & & $0.47^{*}$ & -0.15 & $0.50^{*}$ & & $0.58^{*}$ & -0.10 & $0.50^{*}$ \\
\hline White bread & & $0.34^{\star}$ & & 0.29 & & $0.34^{\star}$ & & $0.37^{\star}$ & -0.14 & $0.45^{\star}$ & & $0.50^{\star}$ & & $0.69^{\star}$ & -0.14 & $0.34^{\star}$ & & $0.48^{\star}$ & -0.11 & $0.49^{*}$ \\
\hline Brown bread & 0.17 & -0.16 & $0.31^{*}$ & -0.17 & $0.36^{*}$ & -0.18 & $0.34^{\star}$ & -0.12 & $0.31^{*}$ & $-0.33^{*}$ & 0.28 & $-0.39^{\star}$ & & $-0.49^{\star}$ & $0.33^{*}$ & -0.12 & $0.38^{*}$ & -0.17 & $0.31^{\star}$ & -0.29 \\
\hline $\begin{array}{l}\text { Baked beans/lentils/soya } \\
\text { mince... }\end{array}$ & 0.19 & $0.37^{\star}$ & 0.24 & 0.22 & 0.27 & $0.33^{*}$ & 0.20 & 0.24 & 0.26 & 0.17 & 0.21 & 0.13 & 0.20 & & 0.28 & 0.25 & 0.23 & 0.15 & 0.19 & 0.15 \\
\hline $\begin{array}{l}\text { Chips/fried or roasted } \\
\text { potatoes }\end{array}$ & & $0.54^{*}$ & -0.10 & $0.45^{*}$ & & $0.54^{*}$ & & $0.43^{*}$ & & $0.53^{*}$ & -0.13 & $0.51^{*}$ & & $0.55^{\star}$ & & $0.59^{*}$ & -0.23 & $0.35^{*}$ & & $0.40^{*}$ \\
\hline Other potatoes & 0.17 & 0.14 & 0.25 & 0.22 & 0.20 & 0.22 & 0.24 & 0.24 & 0.23 & & 0.29 & & 0.20 & 0.16 & 0.21 & 0.23 & $0.38^{\star}$ & $0.35^{\star}$ & 0.22 & 0.11 \\
\hline $\begin{array}{l}\text { Green cooked veg- } \\
\text { etables }\end{array}$ & $0.67^{\star}$ & & $0.68^{*}$ & & $0.68^{*}$ & & $0.79^{*}$ & & $0.64^{*}$ & & $0.67^{\star}$ & & $0.72^{*}$ & & $0.70^{*}$ & & $0.72^{*}$ & & $0.56^{*}$ & -0.16 \\
\hline Other vegetables & $0.59^{*}$ & 0.12 & $0.52^{\star}$ & & $0.63^{*}$ & & $0.75^{*}$ & & $0.57^{\star}$ & & $0.61^{\star}$ & & $0.75^{*}$ & 0.12 & $0.59^{*}$ & & $0.68^{*}$ & & $0.54^{*}$ & -0.16 \\
\hline Salads & 0.28 & -0.12 & $0.41^{*}$ & & $0.38^{*}$ & & $0.30^{*}$ & -0.17 & $0.41^{*}$ & -0.10 & $0.44^{*}$ & & $0.48^{*}$ & & $0.38^{*}$ & & $0.49^{*}$ & & $0.40^{*}$ & \\
\hline Fresh fruits & $0.37^{\star}$ & & $0.45^{*}$ & & $0.43^{*}$ & & $0.44^{\star}$ & & $0.38^{*}$ & & $0.40^{*}$ & -0.11 & $0.59^{\star}$ & 0.16 & $0.38^{*}$ & & $0.46^{*}$ & & $0.45^{*}$ & -0.11 \\
\hline Soft drinks & -0.14 & 0.19 & -0.11 & $0.36^{*}$ & & $0.38^{*}$ & & $0.42^{*}$ & -0.12 & 0.29 & & $0.32^{\star}$ & -0.14 & 0.29 & & $0.31^{*}$ & & 0.29 & & $0.43^{*}$ \\
\hline Low-energy soft drinks & -0.19 & & -0.11 & 0.13 & & 0.15 & -0.13 & & -0.13 & 0.13 & -0.11 & 0.17 & & 0.15 & & 0.12 & & 0.14 & & 0.29 \\
\hline Fruit juice/squash & & $0.36^{*}$ & & $0.36^{*}$ & 0.13 & $0.30^{*}$ & & 0.21 & & 0.25 & & 0.24 & & 0.24 & & 0.22 & 0.15 & 0.27 & & $0.37^{\star}$ \\
\hline Unsweetened fruit juice & 0.22 & & 0.24 & & 0.14 & & 0.26 & & 0.22 & -0.10 & 0.24 & & 0.23 & & 0.22 & & 0.19 & & 0.21 & \\
\hline Lard/dripping & -0.16 & & -0.10 & & & & & 0.13 & & 0.23 & & 0.25 & & & -0.18 & & -0.15 & 0.18 & -0.17 & 0.16 \\
\hline Olive oil & $0.34^{\star}$ & -0.16 & $0.35^{\star}$ & -0.18 & $0.40^{*}$ & -0.15 & 0.26 & -0.15 & $0.34^{*}$ & -0.18 & $0.32^{*}$ & -0.15 & $0.35^{*}$ & -0.12 & $0.36^{\star}$ & -0.15 & $0.38^{*}$ & -0.16 & $0.32^{\star}$ & $-0.31^{*}$ \\
\hline Vegetable oil & 0.13 & $0.52^{\star}$ & 0.18 & $0.37^{\star}$ & 0.16 & $0.32^{*}$ & & $0.32^{\star}$ & 0.12 & $0.43^{\star}$ & 0.13 & $0.37^{*}$ & & $0.35^{*}$ & 0.24 & $0.46^{*}$ & & 0.23 & 0.11 & 0.27 \\
\hline Butter & & 0.16 & -0.20 & & & 0.24 & & & 0.12 & $0.30^{*}$ & 0.16 & 0.27 & & 0.11 & & 0.24 & 0.11 & 0.20 & & \\
\hline Margarine & & & 0.13 & & 0.15 & & & 0.27 & -0.13 & & & & -0.11 & & -0.11 & 0.12 & & & & \\
\hline Low-fat margarine & 0.16 & & 0.15 & & & & & & & & & & & & & -0.12 & & & 0.11 & \\
\hline $\begin{array}{l}\text { Supplements (vitamins, } \\
\text { minerals and fish oils) }\end{array}$ & & & & -0.12 & 0.24 & -0.17 & 0.16 & & 0.22 & & 0.21 & & & & 0.12 & & 0.10 & & & -0.12 \\
\hline
\end{tabular}

$\mathrm{H}$, Healthy pattern; UnH, Unhealthy pattern.

* The values exceed $0 \cdot 30$, considered sufficiently strong to be retained. 
Table 3. Consistency of the dietary patterns from 5 to 13 years, measured by Tucker's congruence coefficient $(\varphi)$

\begin{tabular}{|c|c|c|c|c|c|c|c|c|c|c|}
\hline & 5 years & 6 years & 7 years & 8 years & 9 years* & 9 years $†$ & 10 years & 11 years & 12 years & 13 years \\
\hline \multicolumn{11}{|l|}{$\varphi_{\text {Healthy }}$} \\
\hline 5 years & $x$ & 0.73 & 0.67 & 0.68 & 0.72 & 0.71 & 0.72 & 0.70 & 0.67 & 0.74 \\
\hline 6 years & & $\times$ & 0.67 & 0.66 & 0.67 & 0.68 & 0.68 & 0.67 & 0.65 & 0.76 \\
\hline 7 years & & & $\times$ & 0.68 & 0.70 & 0.70 & 0.66 & 0.68 & 0.67 & 0.76 \\
\hline 8 years & & & & $\times$ & 0.70 & 0.70 & 0.72 & 0.66 & 0.67 & 0.73 \\
\hline 9 years* & & & & & $\times$ & 0.74 & 0.70 & 0.70 & 0.66 & 0.74 \\
\hline 9 years $†$ & & & & & & $\times$ & 0.70 & 0.69 & 0.68 & 0.74 \\
\hline 10 years & & & & & & & $\times$ & 0.68 & 0.69 & 0.75 \\
\hline 11 years & & & & & & & & $\times$ & 0.65 & 0.74 \\
\hline 12 years & & & & & & & & & $\times$ & 0.75 \\
\hline 13 years & & & & & & & & & & $\times$ \\
\hline \multicolumn{11}{|l|}{$\varphi$ Unhealthy } \\
\hline 5 years & $x$ & 0.75 & 0.68 & 0.68 & 0.67 & 0.65 & 0.63 & 0.76 & 0.71 & 0.67 \\
\hline 6 years & & $\times$ & 0.72 & 0.73 & 0.71 & 0.70 & 0.68 & 0.78 & 0.76 & 0.73 \\
\hline 7 years & & & $\times$ & 0.68 & 0.65 & 0.65 & 0.62 & 0.72 & 0.72 & 0.67 \\
\hline 8 years & & & & $\times$ & 0.66 & 0.65 & 0.64 & 0.73 & 0.75 & 0.68 \\
\hline 9 years* & & & & & $\times$ & 0.71 & 0.64 & 0.71 & 0.70 & 0.65 \\
\hline 9 years $\dagger$ & & & & & & $\times$ & 0.65 & 0.70 & 0.70 & 0.67 \\
\hline 10 years & & & & & & & $\times$ & 0.68 & 0.70 & 0.65 \\
\hline 11 years & & & & & & & & $\times$ & 0.77 & 0.71 \\
\hline 12 years & & & & & & & & & $\times$ & 0.73 \\
\hline 13 years & & & & & & & & & & $\times$ \\
\hline
\end{tabular}

* Based on the original sample of 302 children.

$\dagger$ Based on the sample of 342 children.

and/or energy intakes rather than dietary choice ${ }^{(11-15)}$. Our data highlight the importance of repeat measures, not only to establish trends, but also to categorise accurately. A onceonly measure of dietary choice, however carefully obtained, is of limited value. The focus has been set more recently on patterns $^{(2,9,10,16,17)}$, but so far only one other study has assessed the consistency of dietary patterns as opposed to dietary constituents in children ${ }^{(16)}$.

The present study has strengths and limitations. To our knowledge, it is the first to have repeatedly recorded the dietary choices of children over a protracted period. The FFQ response rates were high and maintained over the nine time points sampled, enabling us to apply longitudinal analysis to the data and assess reliability of the measurements. Care had been taken at recruitment to ensure random sampling of the population and the uniformity of age needed to resolve age-related change (trend). A true limitation of the present study is its restriction (98\%) to White Caucasian children, and our simple factor solution (two dietary patterns only) could partly be explained by the homogeneity of the

Table 4. Consistency of the individual dietary $z$-scores from 5 to 13 years, measured by Pearson's correlation coefficient ${ }^{\star}(r)$

\begin{tabular}{|c|c|c|c|c|c|c|c|c|c|c|}
\hline & 5 years & 6 years & 7 years & 8 years & 9 years $†$ & 9 years $\ddagger$ & 10 years & 11 years & 12 years & 13 years \\
\hline \multicolumn{11}{|l|}{$r_{\text {Healthy }}$} \\
\hline 5 years & $x$ & 0.69 & 0.68 & 0.53 & 0.56 & 0.55 & 0.49 & 0.52 & 0.54 & 0.46 \\
\hline 6 years & & $\times$ & 0.71 & 0.62 & 0.67 & 0.66 & 0.58 & 0.56 & 0.56 & 0.53 \\
\hline 7 years & & & $\times$ & 0.68 & 0.67 & 0.67 & 0.53 & 0.53 & 0.52 & 0.52 \\
\hline 8 years & & & & $\times$ & 0.68 & 0.69 & 0.62 & 0.56 & 0.65 & 0.56 \\
\hline 9 years $\dagger$ & & & & & $\times$ & 1.00 & 0.63 & 0.66 & 0.59 & 0.57 \\
\hline 9 years $\ddagger$ & & & & & & $\times$ & 0.64 & 0.63 & 0.62 & 0.58 \\
\hline 10 years & & & & & & & $\times$ & 0.60 & 0.59 & 0.51 \\
\hline 11 years & & & & & & & & $\times$ & 0.66 & 0.60 \\
\hline 12 years & & & & & & & & & $\times$ & 0.70 \\
\hline 13 years & & & & & & & & & & $\times$ \\
\hline \multicolumn{11}{|l|}{$r_{\text {Unhealthy }}$} \\
\hline 5 years & $x$ & 0.61 & 0.52 & 0.58 & 0.43 & 0.42 & 0.39 & 0.40 & 0.45 & 0.33 \\
\hline 6 years & & $\times$ & 0.65 & 0.66 & 0.58 & 0.57 & 0.46 & 0.51 & 0.52 & 0.47 \\
\hline 7 years & & & $\times$ & 0.61 & 0.61 & 0.60 & 0.47 & 0.49 & 0.49 & 0.42 \\
\hline 8 years & & & & $\times$ & 0.68 & 0.68 & 0.53 & 0.52 & 0.60 & 0.54 \\
\hline 9 years $†$ & & & & & $\times$ & 1.00 & 0.61 & 0.62 & 0.54 & 0.49 \\
\hline 9 yearsł & & & & & & $\times$ & 0.63 & 0.61 & 0.55 & 0.50 \\
\hline 10 years & & & & & & & $\times$ & 0.57 & 0.53 & 0.46 \\
\hline 11 years & & & & & & & & $\times$ & 0.63 & 0.59 \\
\hline 12 years & & & & & & & & & $\times$ & 0.66 \\
\hline 13 years & & & & & & & & & & $\times$ \\
\hline
\end{tabular}

* All $P<0.001$.

† Based on the original sample of 302 children.

$\ddagger$ Based on the sample of 342 children. 


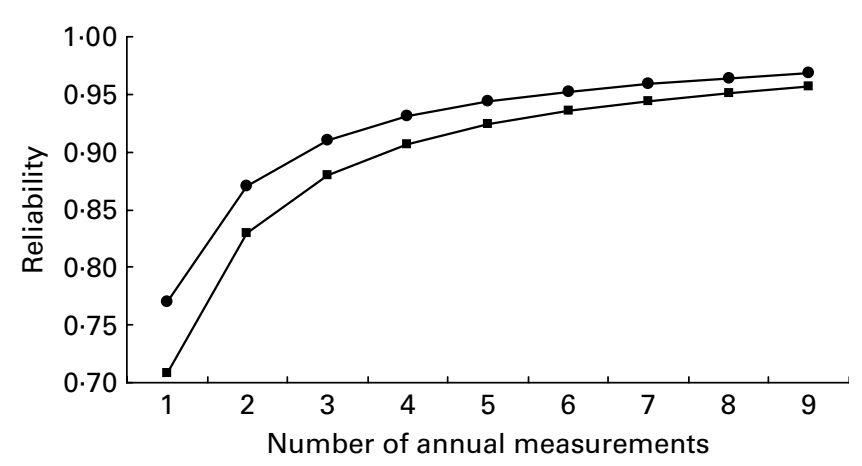

Fig. 1. Mean reliability of the dietary $z$-scores according to the factor and number of consecutive annual measurements. $\longrightarrow-$, Healthy $z$-score; $\longrightarrow$, unhealthy $z$-score.

sample. Had we recruited a more multi-cultural cohort, the results might have been more generalisable but also more 'complex' in terms of factor solution owing to more dietary patterns that emerged. No information regarding portion sizes was available, and, as with all such studies, the possibility of misreporting cannot be ruled out.

The variance in dietary choice explained by the factors retained is inversely dependent on the number of variables included in the analysis. The estimate of approximately $16 \%$ in this analysis appears to be small but is close to what has been reported in other studies ${ }^{(2,16-18)}$. McCann et al. ${ }^{(20)}$ suggested that the proportion of variance explained might not be as important as the interpretability of the factors in relation to disease risk, and that attempts to increase the variance explained by removing the least meaningful items could weaken rather than strengthen the association with disease outcome. Kim \& Mueller ${ }^{(21)}$ warned that deletion of items in order to simplify the factorial structure may even lead to erroneous conclusions.

The consistency in the dietary $z$-scores among individuals in our cohort is comparable with that reported by Northstone \& Emmett $^{(16)}$, who studied the dietary patterns of a large cohort of children ( $n$ 6177) based on four occasions between 3 and 9 years. Although the authors reported close tracking of the dietary $z$-scores year-on-year ( $r$ approximately $0 \cdot 61$ ), they did not provide a true measure of congruence for their patterns, despite observing changes in their factorial solution over time (notably the emergence of a new pattern). In a 1-year follow-up of adolescents ( $n$ 216), Li \& Wang ${ }^{(9)}$ found weaker tracking of their dietary scores involving three factors $(r 0.47,0.36$ and 0.31$)$, but, again, the degree of congruence between baseline and follow-up factorial solutions was not reported.

We found, not unexpectedly, that repeat measurements over long intervals were less consistent than the year-onyear comparisons. Others have reported the same ${ }^{(8,14)}$, and it suggests that children's dietary habit may change gradually over time. Perhaps parents have less influence over what their child consumes as they approach their teenage years. Newby and colleagues suggested that changes in the food supply, and in what is perceived as 'healthy', could contribute over time ${ }^{(22)}$.

More children witnessed deterioration than improvement over time in the quality of their diet. Mannino et al. ${ }^{(12)}$ reported much the same among girls between 5 and 9 years. Despite close tracking of their dietary choices across middle childhood, fewer girls in Mannino's study met the dietary recommendation for most food groups at 9 years than had done so at 5 years. As Singer et al. ${ }^{(13)}$, Mannino concluded that eating patterns are established early in life, and that early review of young families may help ensure healthy eating habits later. Reliability in our dietary $z$-scores increased with the number of repeated measurements and was correspondingly higher than that reported by $\mathrm{Hu}$ et $a l^{(17)}$ based on two repeats for a 'Prudent' and a 'Western' diet (70 and 67\%, respectively).

It is important that patterns identified by factor analysis be interpreted as a set, in order to understand the overall structure of an individual's diet, for it is entirely possible to score high (or low) on both 'Healthy' and 'Unhealthy' factors. A high $z$-score for the Healthy pattern does not preclude a high $z$-score for the Unhealthy pattern as well, and the possibility has not always been made clear in studies using factor analysis. It is also possible that opposing dietary patterns be correlated, and systematic use of the orthogonal (Varimax) rotation in the extraction of factors should be reconsidered on this account, particularly in the context of association with disease risk.

\section{Conclusions/future research}

Repeat measurements are needed to ensure the reliability of dietary data. Dietary habits appear to be established early in life and are broadly retained throughout childhood. Notwithstanding, more children appear to experience a deterioration in their diet over childhood than improvement. Several studies have suggested that dietary patterns can predict future health $^{(23-29)}$, and early assessment of dietary habits seems crucial to disease prevention. Members of the Early Bird cohort are approaching adulthood, and the links between dietary patterns of childhood, body composition, physical activity and metabolic health will be key analyses when they do.

\section{Acknowledgements}

We are indebted to the parents, children and their schools for their cooperation in conducting the present study. The authors thank Karen Brookes and Val Morgan for their assistance with the data collection. The EarlyBird Diabetes Study is currently funded by Bright Futures Trust, Child Growth Foundation, Kirby Laing Foundation and Nestlé. None had any involvement in the study design, collection or analysis of data, interpretation of findings, or writing of the manuscript. The authors declare no conflict of interest. A. E. F. was responsible for the processing and analysis of the data, interpretation of the results and writing of the manuscript. J. H., B. S. M. and A. N. J. participated in the data collection and statistical interpretation of the results. L. D. V. participated in the planning of the study. T. J. W. was the guarantor of the study, and participated in the interpretation of the results and writing of the manuscript. 


\section{References}

1. DeFronzo RA (2010) Insulin resistance, lipotoxicity, type 2 diabetes and atherosclerosis: the missing links. Diabetologia 53, $1270-1287$.

2. Mikkila V, Rasanen L, Raitakari OT, et al. (2004) Longitudinal changes in diet from childhood into adulthood with respect to risk of cardiovascular diseases: The Cardiovascular Risk in Young Finns Study. Eur J Clin Nutr 58, 1038-1045.

3. Mikkila V, Rasanen L, Raitakari OT, et al. (2005) Consistent dietary patterns identified from childhood to adulthood: the cardiovascular risk in Young Finns Study. BrJ Nutr 93, 923-931.

4. Martinez ME, Marshall JR \& Sechrest L (1998) Invited Commentary: factor analysis and the search for objectivity. Am J Epidemiol 148, 17-19.

5. Voss LD, Kirkby J, Metcalf BS, et al. (2003) Preventable factors in childhood that lead to insulin resistance, diabetes mellitus and the metabolic syndrome: the EarlyBird diabetes study 1. J Pediatr Endocrinol Metab 16, 1211-1224.

6. Hammond J, Nelson M, Chinn S, et al. (1993) Validation of a food frequency questionnaire for assessing dietary intake in a study of coronary heart disease risk factors in children. Eur J Clin Nutr 47, 242-250.

7. Catell RB (1966) The scree test for the number of factors. Multivar Behav Res 1, 245-276.

8. Tucker LR (1951) A method for synthesis of factor analysis studies. Personnel Research Section Report no. 984 Washington, DC: Department of the Army.

9. Li J \& Wang Y (2008) Tracking of dietary intake patterns is associated with baseline characteristics of urban lowincome African-American adolescents. J Nutr 138, 94-100.

10. Cutler GJ, Flood A, Hannan P, et al. (2009) Major patterns of dietary intake in adolescents and their stability over time. J Nutr 139, 323-328.

11. Zive MM, Berry CC, Sallis JF, et al. (2002) Tracking dietary intake in white and Mexican-American children from age 4 to 12 years. J Am Diet Assoc 102, 683-689.

12. Mannino ML, Lee Y, Mitchell DC, et al. (2004) The quality of girls' diets declines and tracks across middle childhood. Int J Behav Nutr Phys Act 1, 5.

13. Singer MR, Moore LL, Garrahie EJ, et al. (1995) The tracking of nutrient intake in young children: the Framingham Children's Study. Am J Public Health 85, 1673-1677.

14. Wang Y, Bentley ME, Zhai F, et al. (2002) Tracking of dietary intake patterns of Chinese from childhood to adolescence over a six-year follow-up period. J Nutr 132, 430-438.

15. Stein AD, Shea S, Basch CE, et al. (1991) Variability and tracking of nutrient intakes of preschool children based on multiple administrations of the 24-hour dietary recall. $\mathrm{Am} \mathrm{J}$ Epidemiol 134, 1427-1437.
16. Northstone K \& Emmett PM (2008) Are dietary patterns stable throughout early and mid-childhood? A birth cohort study. Br J Nutr 100, 1069-1076.

17. Hu FB, Rimm E, Smith-Warner SA, et al. (1999) Reproducibility and validity of dietary patterns assessed with a food-frequency questionnaire. Am J Clin Nutr 69, 243-249.

18. Slattery ML, Boucher KM, Caan BJ, et al. (1998) Eating patterns and risk of colon cancer. Am J Epidemiol 148, 4-16.

19. Paradis AM, Perusse L \& Vohl MC (2006) Dietary patterns and associated lifestyles in individuals with and without familial history of obesity: a cross-sectional study. Int J Behav Nutr Phys Act 3, 38.

20. McCann SE, Marshall JR, Brasure JR, et al. (2001) Analysis of patterns of food intake in nutritional epidemiology: food classification in principal components analysis and the subsequent impact on estimates for endometrial cancer. Public Health Nutr 4, 989-997.

21. Kim J-O \& Mueller CW (1978) Introduction to factor analysis: what it is and how to do it? Sage University Paper Series on Quantitative Applications in the Social Sciences, pp. 7-13 Newbury Park, CA: University of California Press.

22. Guallar-Castillón P, Rodríguez-Artalejo $\mathrm{F}$, Tormo MJ, et al. (2010) Major dietary patterns and risk of coronary heart disease in middle-aged persons from a Mediterranean country: The EPIC-Spain cohort study. Nutr Metab Cardiovasc Dis (Epublication ahead of print version 11 August 2010).

23. Mikkila V, Rasanen L, Raitakari OT, et al. (2007) Major dietary patterns and cardiovascular risk factors from childhood to adulthood. The Cardiovascular Risk in Young Finns Study. Br J Nutr 98, 218-225.

24. Mikkila V, Rasanen L, Laaksonen MM, et al. (2009) Long-term dietary patterns and carotid artery intima media thickness: the Cardiovascular Risk in Young Finns Study. Br J Nutr 102, 1507-1512.

25. Denova-Gutierrez E, Castanon S, Talavera JO, et al. (2010) Dietary patterns are associated with metabolic syndrome in an urban Mexican population. J Nutr 140, 1855-1863.

26. McNaughton SA, Mishra GD \& Brunner EJ (2009) Food patterns associated with blood lipids are predictive of coronary heart disease: the Whitehall II study. Br J Nutr 102, 619-624.

27. Nettleton JA, Steffen LM, Mayer-Davis EJ, et al. (2006) Dietary patterns are associated with biochemical markers of inflammation and endothelial activation in the Multi-Ethnic Study of Atherosclerosis (MESA). Am J Clin Nutr $\mathbf{8 3}$ 1369-1379.

28. Sadakane A, Tsutsumi A, Gotoh T, et al. (2008) Dietary patterns and levels of blood pressure and serum lipids in a Japanese population. J Epidemiol 18, 58-67.

29. Ambrosini GL, Huang RC, Mori TA, et al. (2010) Dietary patterns and markers for the metabolic syndrome in Australian adolescents. Nutr Metab Cardiovasc Dis 20, 274-283. 